\title{
Quantifying Cortical EEG Responses to TMS in (Un)consciousness
}

\author{
Simone Sarasso', Mario Rosanova ${ }^{1,2}$, Adenauer G. Casali ${ }^{1,3}$, \\ Silvia Casarotto', Matteo Fecchio', Melanie Boly,5, \\ Olivia Gosseries ${ }^{4,5}$, Giulio Tononi ${ }^{5}$, Steven Laureys ${ }^{4}$, and Marcello Massimini ${ }^{1,6}$
}

\begin{abstract}
We normally assess another individual's level of consciousness based on her or his ability to interact with the surrounding environment and communicate. Usually, if we observe purposeful behavior, appropriate responses to sensory inputs, and, above all, appropriate answers to questions, we can be reasonably sure that the person is conscious. However, we know that consciousness can be entirely within the brain, even in the absence of any interaction with the external world; this happens almost every night, while we dream. Yet, to this day, we lack an objective, dependable measure of the level of consciousness that is independent of processing sensory inputs and producing appropriate motor outputs. Theoretically, consciousness is thought to require the joint presence of functional integration and functional differentiation, otherwise defined as brain complexity. Here we review a series of recent studies in which Transcranial Magnetic Stimulation combined with electroencephalography (TMS/EEG) has been employed to quantify brain complexity in wakefulness and during physiological (sleep), pharmacological (anesthesia) and pathological (brain injury) loss of consciousness. These studies invariably show that the complexity of the cortical response to TMS collapses when consciousness is lost during deep sleep, anesthesia and vegetative state following severe brain injury, while it recovers when consciousness resurges in wakefulness, during dreaming, in the minimally conscious state or locked-in syndrome. The present paper will also focus on how this approach may contribute to unveiling the pathophysiology of disorders of consciousness affecting brain-injured patients. Finally, we will underline some crucial methodological aspects concerning TMS/EEG measurements of brain complexity.
\end{abstract}

\section{Keywords}

TMS/EEG, brain complexity, bistability, sleep, anesthesia, coma

\section{Introduction}

Everyone knows what consciousness is: it is what vanishes when we fall into dreamless sleep and reappears when we wake up or when we dream ${ }^{1}$ - in other words, it is synonymous with experience. Assessing consciousness is often straightforward: if we see purposeful, intelligent behavior in a person, we assume she or he is conscious. If in doubt, as when someone is resting with eyes closed, we can ask the person; if the person answers that she or he was thinking or daydreaming, we infer she or he was conscious. But at times matters are less clear: someone fast asleep shows no purposeful activity and will not respond to questions, yet she or he may be dreaming. Similarly, some patients with brain damage may be behaviorally unresponsive and thus judged clinically unconscious, yet they may be able to generate brain signals indicating they understood a question or a command. ${ }^{2,3}$ In general, the problem is that while we assess the level of consciousness based on an individual's ability to connect and respond to the external environment, these features are not necessary for consciousness. Yet, to this day, we do not have a scientifically well-grounded measure of

\footnotetext{
'Department of Biomedical and Clinical Sciences "Luigi Sacco," University of Milan, Milan, Italy

${ }^{2}$ Fondazione Europea di Ricerca Biomedica, Milan, Italy

${ }^{3}$ Faculty of Medicine Clinics Hospital, University of São Paulo, 05403-000, São Paulo, Brazil

${ }^{4}$ Coma Science Group, Cyclotron Research Centre and Neurology Department, University and University Hospital of Liège, Liège, Belgium ${ }^{5}$ Department of Psychiatry, University of Wisconsin, Madison, WI, USA ${ }^{6}$ Istituto di Ricovero e Cura a Carattere Scientifico, Fondazione Don Carlo Gnocchi, Milan, Italy
}

\section{Corresponding Author:}

Marcello Massimini, Department of Biomedical and Clinical Sciences "Luigi Sacco," University of Milan, LITA building, Via G.B. Grassi 74, 20157 Milan, Italy.

Email: marcello.massimini@unimi.it

Full-color figures are available online at http://eeg.sagepub.com 
the level of consciousness that is independent of processing sensory inputs and producing appropriate motor outputs.

Neuroscience is certainly making progress in identifying the neural correlates of consciousness. While many of the proposed neural substrates of consciousness undoubtedly have heuristic value, empirical evidence still does not provide criteria for necessity and sufficiency. For example, measurements performed during seizures ${ }^{4}$ where subjects are unconscious and unresponsive despite increased brain metabolism have suggested that the overall levels of brain activity may not be a reliable marker of the presence of consciousness. Along the same lines, positron emission tomography measurements have shown that brain-injured patients can recover consciousness from a vegetative state, without necessarily increasing their brain metabolic rates. ${ }^{5}$ On the other hand, the hypothesis that the level of consciousness could be critically determined by the power/synchronization of spontaneous, fast frequency oscillations in the thalamocortical system has been questioned by recent measurements. Indeed, this hypothesis fails to explain the loss of consciousness (LOC) observed during non-rapid eye movement (NREM) sleep, propofol anesthesia, and generalized tonicclonic seizures, where hypersynchronous broadband oscillations can be observed. ${ }^{6}$ As a consequence, even apparently simple questions like "Why does consciousness fade during early NREM sleep?" and "Why does it resume during dreaming?" have been (and still are) unanswered, thus pointing to the need for robust empirical studies complemented by a self-consistent, general, and parsimonious theoretical approach.

A recently proposed theory suggests that consciousness requires the coexistence of integration and information in thalamocortical networks - otherwise defined as brain complexity. $^{7-10}$ Neurophysiologically, this depends on the ability of neural elements to engage in complex activity patterns that are, at once, distributed within a system of interacting cortical areas (integrated) and differentiated in space and time (informationrich). In brief, to sustain consciousness, the thalamocortical system is endowed with the following 2 properties: (1) information - the system has a large repertoire of available states so that, when it enters a particular state through causal interactions among its elements, it rules out a large number of alternative states and therefore generates a large amount of information; (2) integration - the system cannot be decomposed into a collection of causally independent subsystems so that, when it enters a particular state, it generates information as a whole (ie, integrated information, above and beyond the information generated independently by its parts).

Although integrated information can be measured exactly only in small simulated systems, the theory makes clear-cut predictions that can be addressed experimentally at least at a gross level. As an example, the fading of consciousness during early NREM sleep (one of the basic unanswered questions above) should be associated with either a reduction of integration within the main thalamocortical complex (eg, it could break down into causally independent modules) or a reduction of information (the repertoire of available states might shrink),

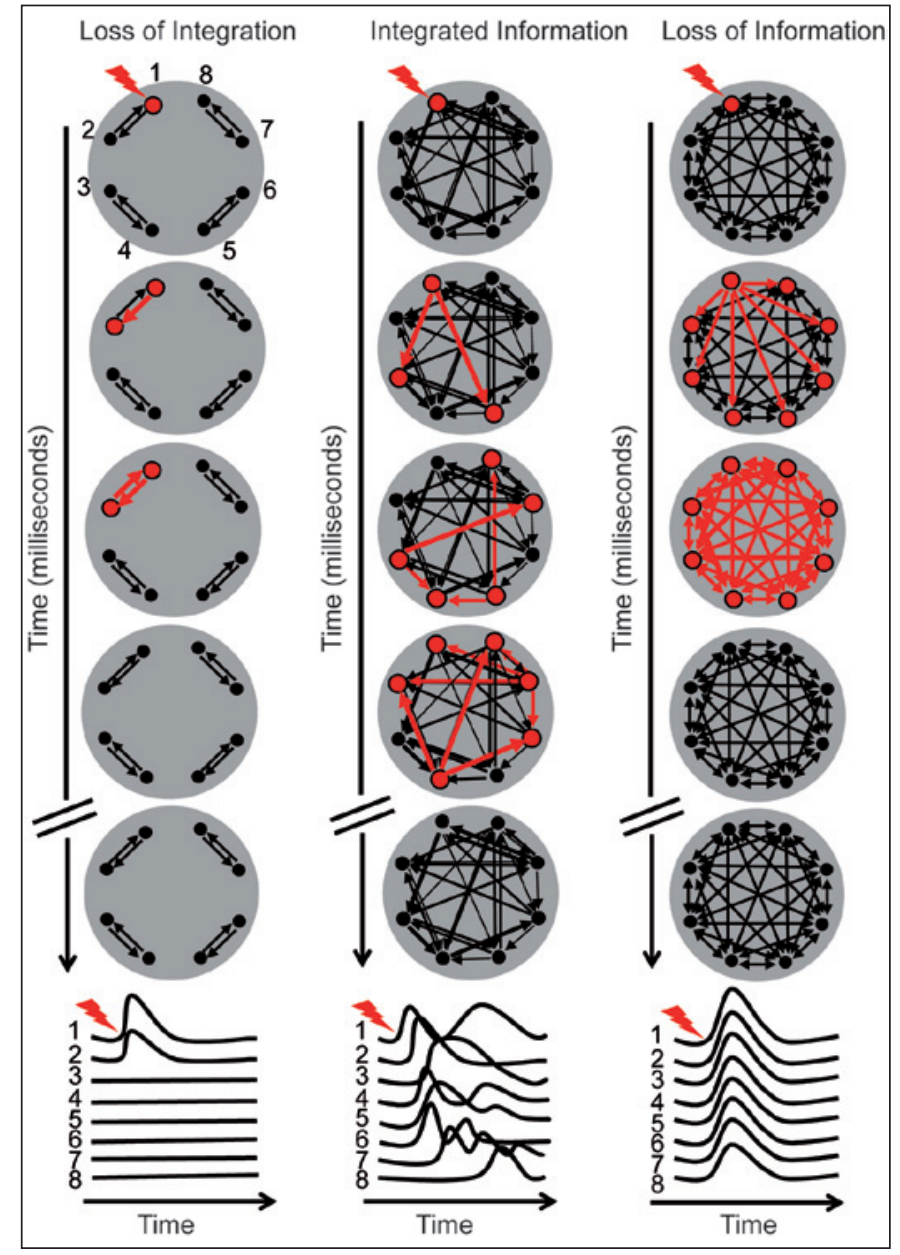

Figure I. An empirical approximation of a theoretical measure. (left) Loss of integration: perturbing the first element results in a short-leaved, local activation involving only the element connected to the perturbed one. (center) Integrated information: only in this case, perturbing the first element results in a long-lasting, widespread yet complex response involving different elements at different time intervals. (right) Loss of information/differentiation: perturbing the first element results in a widespread activation involving all the elements at the same time and then rapidly vanishing.

or both. In a word, integrated information should be high when consciousness is present and low whenever consciousness is lost. Practically, a straightforward way to gauge the conjoint presence of integration and information in real brains involves directly probing the cerebral cortex (to avoid possible subcortical filtering and gating) by employing a perturbational approach (thus testing causal interactions rather than temporal correlations) and examining to what extent cortical regions can interact as a whole (integration) to produce differentiated responses (information; Figure 1). Guided by these principles, over the past 10 years we have employed a combination of navigated transcranial magnetic stimulation (TMS) and high-density electroencephalography (hd-EEG) to measure noninvasively 
and with good spatiotemporal resolution the brain response to the direct perturbation of different subsets of cortical areas. ${ }^{11}$

\section{TMS-EEG Studies}

At first, we systematically tested the aforementioned theoretical predictions in a series of controlled experiments aimed at measuring the cortico-cortical EEG evoked responses to a direct TMS perturbation. Using a 60-channel TMS-compatible EEG amplifier, we recorded TMS-evoked brain responses in healthy subjects whose level of consciousness was experimentally manipulated under both physiological ${ }^{12-14}$ and pharmacological ${ }^{15}$ conditions, and compared the obtained responses to those recorded during wakefulness. We then extended these initial observations to the study of pathological conditions in which consciousness was impaired and performed TMS/EEG measurements in brain-injured patients with a broad spectrum of clinical diagnoses, ranging from the vegetative state (VS)/ unresponsive wakefulness syndrome (UWS) to the minimally conscious state (MCS) and locked-in syndrome (LIS). ${ }^{16}$

\section{TMS/EEG Apparatus}

Stimulation Parameters. Stimulations are performed by means of a figure-of- 8 coil, with a wing diameter of $70 \mathrm{~mm}$, connected to a biphasic stimulator. At least 200 trials are usually collected for each stimulation site. Stimulations are delivered with an interstimulus interval jittering randomly between 2000 and $3000 \mathrm{~ms}(0.3-0.5 \mathrm{~Hz})$, at an intensity ranging from $90 \mathrm{~V} / \mathrm{m}$ up to $160 \mathrm{~V} / \mathrm{m}$ on the cortical surface; TMS pulses within this range are largely above the threshold $(50 \mathrm{~V} / \mathrm{m})$ for an EEG response. ${ }^{16-18}$

EEG Recordings. TMS-evoked EEG activity is recorded by means of a 60 carbon electrodes cap and a specifically designed TMScompatible amplifier (Nexstim Ltd, Helsinki, Finland). The artifact induced by TMS is gated, and saturation of the amplifier is avoided by means of a proprietary sample-and-hold circuit that keeps the analog output of the amplifier constant from $100 \mu \mathrm{s}$ pre- to 2 ms poststimulus. ${ }^{19}$ To further optimize TMS compatibility, the impedance at all electrodes is kept below $3 \mathrm{~K} \Omega$. The EEG signals, referenced to an additional electrode on the forehead, are filtered $(0.1-500 \mathrm{~Hz})$ and sampled at $1450 \mathrm{~Hz}$ with 16-bit resolution. Two extra sensors are used to record the electrooculogram. In most cases, no signs of TMS induced magnetic artifact were detected, and in all cases the EEG signals were artifact-free from $<10$ milliseconds poststimulus.

TMS Targeting. Cortical TMS targets are identified on T1-weighted MR images of the subjects' whole heads. To ensure precision and reproducibility of stimulation a Brain Navigated Stimulation (NBS) system (Nexstim Ltd, Helsinki, Finland) is employed. The NBS device locates (with an error $<1 \mathrm{~mm}$ ) the relative positions of the subject's head and of the TMS coil by means of an optical tracking system. NBS also calculates the distribution and the strength of the intracranial electric field induced by TMS. This computation is based on a spherical model and takes into account the exact shape of the coil and its 3D position and orientation, as well as the overall shape of the subject's head and brain. In this way, the exact location of the maximum electric field on the cortical surface can be monitored in real time. The coordinates of stimulation are then passed to a software aiming tool that ensures the reproducibility of position, direction, and angle of the stimulator throughout a single session as well as across different sessions. ${ }^{18,20}$

\section{NREM Sleep}

In a first series of studies, we investigated the changes in TMSevoked EEG responses during the transition from wakefulness to NREM sleep early in the night, when consciousness fades. ${ }^{12}$ During wakefulness, TMS triggers a sequence of low-amplitude, high-frequency (ranging from 10 to $30 \mathrm{~Hz}$ ) waves associated with a differentiated (in both space and time) pattern of cortical activations ${ }^{21}$ that propagate along long-range ipsilateral and transcallosal connections (Figures 2A and 2B, left). Thus, in line with the theoretical requirements, during conscious wakefulness a direct cortical perturbation resulted in complex activity patterns that are, at once, distributed within a system of interacting cortical areas (integrated) and differentiated in space and time (information-rich).

In contrast, during NREM sleep, TMS delivered, with the same stimulation parameters, invariably produces a simple wave of activation that remains localized to the site of stimulation, indicating a breakdown of communication and a loss of integration within thalamocortical networks ${ }^{12}$ (Figure 2A, middle). In other words, when subjects lose consciousness during NREM sleep, TMS triggers a low-frequency wave, associated with a strong initial cortical activation, which does not propagate to connected brain regions and dissipates rapidly. Interestingly, the disappearance of a complex spatiotemporal pattern of cortical activation is not simply due to a reduction of responsiveness of hyperpolarized cortical neurons. In fact, when TMS is applied at progressively higher intensity the initial activation is followed by a larger negative wave that spreads like an oil spot to vast regions of the cortex. Particularly, when TMS is applied over the parietal cortex this wave reaches period-amplitude criteria of a full-fledged sleep slow wave. ${ }^{13}$ Thus, during NREM sleep an increase of the spread of the response (integration) comes with the price of a loss of differentiation (Figure 2A, middle).

Overall, these results demonstrated that during NREM the thalamocortical system, despite being active and reactive, loses its ability to engage in complex activity patterns ${ }^{20,21}$ that are, at once, distributed within a system of interacting cortical areas (integrated) and differentiated in space and time (informationrich); it either breaks down in casually independent modules (loss of integration) or bursts in an explosive response (loss of differentiation/information). 
A

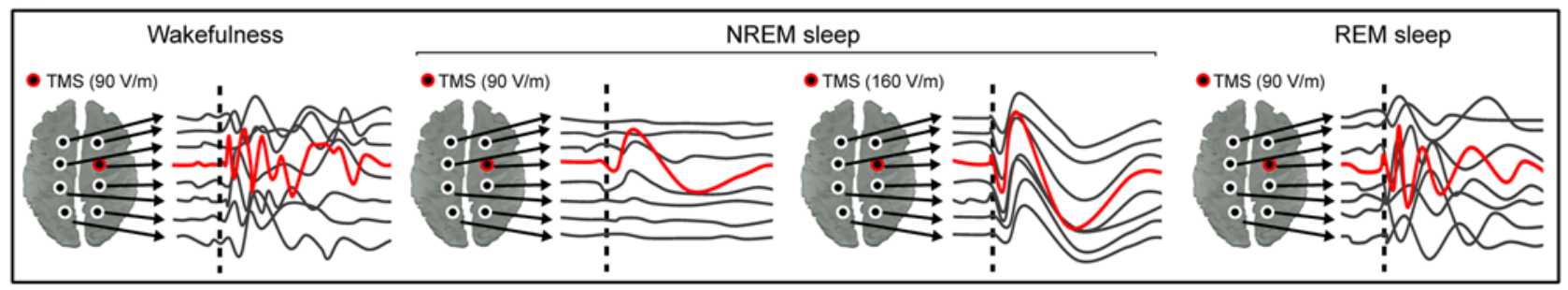

B

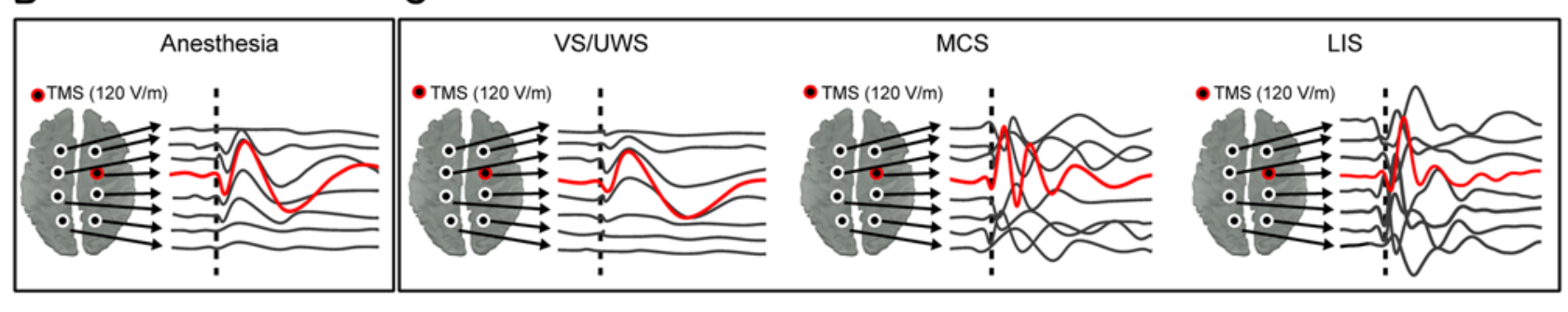

Figure 2. Loss and recovery of integration and information in thalamocortical networks. During wakefulness (A, left), TMS triggers a sustained response made of recurrent waves of activity associated with spatially and temporally differentiated patterns of activation (brain complexity). During NREM sleep (A, middle), anesthesia (B), and vegetative state (C, left) the thalamocortical system, despite being active and reactive, loses its ability to engage in complex activity patterns and either breaks down in casually independent modules (loss of integration) or it bursts in an explosive response (loss of differentiation/information). During REM sleep (A, right) and in MCS (C, middle) and LIS patients (C, right), the TMS response shows a recovery of recurrent waves of activity associated with spatially and temporally differentiated patterns of activation.

\section{Midazolam Anesthesia}

These observations have been replicated in a recent set of experiments where we asked whether cortical effective connectivity would show a breakdown, similar to the one observed in deep NREM sleep, during LOC induced by a pharmacological agent, midazolam, delivered to healthy participants at anesthetic concentrations. ${ }^{15}$ Similar to NREM, we found that, while during wakefulness TMS triggered complex responses involving multiple cortical areas distant from the site of stimulation, during midazolam-induced LOC, TMS evoked a positive-negative response that was initially larger but that remained local (Figure 2B, right). As during NREM sleep, also during midazolam anesthesia, this stereotypical response evolved into a full-fledged slow oscillation when the cerebral cortex was stimulated at higher intensity. ${ }^{22}$

\section{Dreaming}

One of the most striking paradoxes sleep can offer is represented by dreaming. In front of us, we have a person lying down with eyes closed - as during NREM sleep-disconnected from the external environment, almost completely paralyzed and unresponsive. Yet, this person is consciously experiencing something that closely resembles waking activity, and upon awakening is able to verbally report its content. Dreamlike consciousness occurs during various phases of sleep, including sleep onset and late night, especially during rapid eye movement (REM) sleep. Thus, to probe the internal dialogue of the thalamocortical system even in the absence of any behavioral cue, we recorded the TMS-evoked responses during REM sleep in healthy subjects whose consciousness regained in the form of long and vivid dreams. ${ }^{14}$ Consistent with the theoretical predictions, the recovery of conscious experience during REM was accompanied by a widespread and differentiated pattern of cortical activation similar to those observed during wakefulness (Figure 2A, right).

\section{Severe Brain Injury}

Finally, we investigated whether a stereotypical pattern of cortical responses (similar to NREM sleep and midazolam anesthesia) was present also when consciousness was lost due to brain insults. ${ }^{16}$ To do so, we employed TMS/EEG to measure cortical responses at the bedside of VS/UWS patients (ie, awake, but entirely unaware). To minimize the possibility of a misdiagnosis due to fluctuations in behavioral responsiveness, clinical assessment was performed by means of the Coma Recovery Scale-Revised (CRS-R) ${ }^{23}$ repeated 4 times a week, every other day. Invariably, the EEG response to TMS was characterized by a local, stereotypical positive-negative wave similar to those obtained during NREM sleep and anesthesia (Figure 2C, left). Interestingly, the same stimulation performed in noncommunicative brain-injured patients capable of 
purposeful behaviors, such as visual tracking or response to simple commands (therefore diagnosed as MCS), was characterized by regaining a complex spatiotemporal pattern of cortical activations, closely resembling those obtained during wakefulness and REM sleep (Figure 2C, right). This similarity was even more explicit when TMS was performed in LIS patients, who were totally paralyzed except for vertical eye movements through which they could signal their awareness and establish a nonambiguous, functional communication with the external world. Along these lines, in a recent study, Ragazzoni and colleagues ${ }^{24}$ reported reduced cortical connectivity following TMS in VS/UWS patients, as compared to MCS patients and healthy controls.

\section{Consciousness and Complexity: From Theory to Practice}

Theoretical measures based on the idea that consciousness relies on the joint presence of differentiation and integration in neural systems have been proposed over the past decade. ${ }^{1,25,26}$ For example, neural complexity $\left(\mathrm{C}_{\mathrm{N}}\right)^{26}$ is high when small subsets of elements tend to show independence (differentiation), but large subsets show increasing dependence (integration). $\Phi^{1}$ is based on perturbing a system in all possible ways to count the number of different states (differentiation) that can be discriminated through causal interactions within the system as a whole (integration). A related measure, called causal density $\left(\mathrm{C}_{\mathrm{d}}\right),{ }^{25}$ is based on Granger causality and is high if a system's elements are both globally integrated (they predict each other's activity) and differentiated (they contribute to these predictions in different ways). Unlike other measures based on the entropy of spontaneous signals, ${ }^{27}$ these theoretical measures share the insight that the kind of complexity that is relevant for consciousness should be based on the interactivity among different parts of the brain. ${ }^{10,28}$ Unfortunately, the application of $\mathrm{C}_{\mathrm{N}}, \Phi$ and $\mathrm{C}_{\mathrm{d}}$ to actual brains presents substantial practical challenges, such as extraordinary computational demands.

The results outlined in the previous section demonstrated that during LOC the thalamocortical system, despite being active and reactive, loses its ability to engage in complex activity patterns that are, at once, distributed within a system of interacting cortical areas (integrated) and differentiated in space and time (information-rich); it invariably either breaks down in casually independent modules ${ }^{12}$ (loss of integration) or it bursts in an explosive response ${ }^{13}$ (loss of differentiation/ information). The aforementioned TMS/EEG empirical measurements thus provide qualitative support to basic theoretical predictions and pave the way toward a quantification of brain complexity across subjects and conditions, a key requirement for a reliable, unified measurement scale.

\section{The Perturbational Complexity Index}

In order to capture brain complexity by means of a synthetic, quantitative index, we recently developed a theory-driven

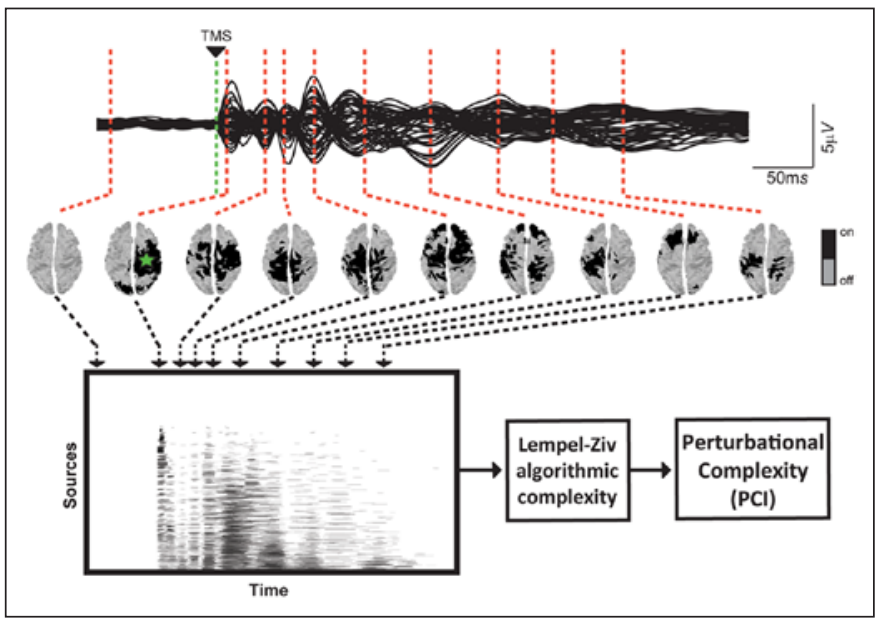

Figure 3. Calculating the $\mathrm{PCl}$. A binary spatiotemporal matrix of significant cortical activation triggered by TMS (green star) is compressed ("zipped”) by Lempel-Ziv algorithmic complexity. Modified from Casali et al. ${ }^{29}$

empirical measure, the so called Perturbational Complexity Index (PCI), which can be practically employed at the patient's bedside. ${ }^{29}$ In agreement with the relevant theoretical requirements for consciousness, PCI gauges the amount of information contained in the integrated response of the thalamocortical system to a direct perturbation. The idea is that the level of consciousness could be estimated empirically by perturbing the cortex ("zapping") to engage distributed interactions and measuring the information content of the ensuing responses by algorithmic compressibility ("zipping"). Operationally, PCI is defined as the normalized Lempel-Ziv algorithmic complexity $^{30}$ of the overall spatiotemporal pattern of significant cortical activation measured by EEG and triggered by a direct cortical perturbation with TMS (Figure 3). In practice, PCI is expected to be low whenever causal interactions among cortical areas are reduced (loss of integration), since the matrix of activation engaged by TMS is spatially restricted; PCI will also be low if many interacting areas react to the perturbation, but they do so in a stereotypical way (loss of differentiation). In this case, the resulting matrix would be large but redundant and could be effectively compressed. It derives that PCI will be high only if the initial perturbation is transmitted to a large set of integrated areas that react in a differentiated way, giving rise to a complex spatiotemporal pattern of deterministic activation that cannot be easily reduced.

We tested PCI on a large data set of TMS-evoked potentials recorded in healthy subjects $(\mathrm{N}=32)$ during wakefulness, dreaming, NREM sleep, and different levels of sedation induced by different anesthetic agents (midazolam, xenon, and propofol), as well as in a group of patients $(\mathrm{N}=20)$ who emerged from coma and recovered consciousness to a variable extent. As shown in Figure 4A, experimentally, PCI was reproducible within and across subjects and depended exclusively 


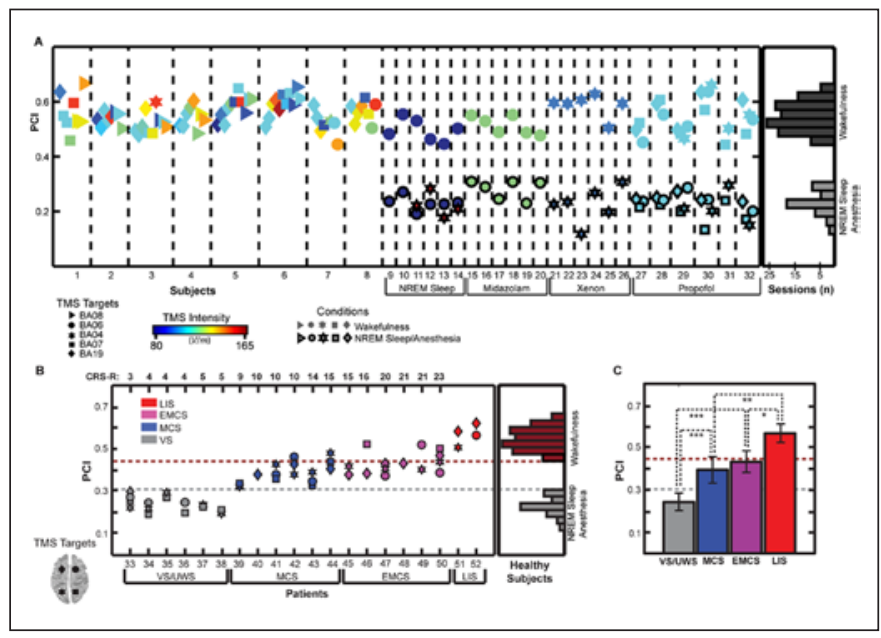

Figure 4. Testing the $\mathrm{PCl}$. (A) $\mathrm{PCl}$ values are shown for $152 \mathrm{TMS}$ sessions collected from 32 healthy subjects. The histograms on the right display the distributions of $\mathrm{PCl}$ across subjects during alert wakefulness (dark gray bars) and loss of consciousness (LOC; light gray bars). $\mathrm{PCl}$ calculated during wakefulness (I 10 sessions) ranged between 0.44 and 0.67 (mean $=0.55 \pm 0.05$ ), whereas the $\mathrm{PCl}$ calculated after LOC (42 sessions) ranged between 0.12 and 0.31 (mean $=0.23 \pm 0.04)$. (B) PCl values are shown for $48 \mathrm{TMS}$ sessions collected from 20 severely brain-injured patients. $\mathrm{PCl}$ followed the level of consciousness (as clinically assessed with CRS-R) progressively increasing from VS/UWS through MCS and recovery of functional communication (EMCS) and attaining levels of healthy awake subjects in LIS. Patient results are directly compared with the ones obtained in healthy individuals. (C) Box plots for $\mathrm{PCl}$ in brain-injured patients are presented with the statistical significance between pairs of conditions (dashed black lines). $* P<.005$. $* * P<$ .0005 . Modified from Casali et al. ${ }^{29}$

on the level of consciousness in all conditions. Specifically, PCI was always high in wakefulness, irrespectively of TMS stimulation site and intensity, but dropped drastically when subjects lost consciousness in NREM sleep, after administration of midazolam, and during general anesthesia with propofol and xenon. In all these conditions, PCI was invariably reduced resulting in a clear-cut distinction between the distributions of the conscious and unconscious states. Crucially, PCI was as low as in NREM sleep and anesthesia in patients with a stable clinical diagnosis of VS/UWS, but was invariably higher in subjects who regained consciousness, including MCS, emerging from MCS (EMCS) and LIS patients (Figure 4B and 4C).

These results indicate that PCI provides an entirely datadriven metric that is reproducible across different conditions (wakefulness, dreaming, LIS, MCS, EMCS, NREM sleep, midazolam sedation, xenon and propofol anesthesia, VS/UWS) and comparable within and across single subjects in the same coordinate space. The main reason for this unprecedented result may reside in the fact that perturbational complexity gauges, at the same time, both the information content and the integration of brain activations. Indeed, PCI combines measures of algorithmic complexity with a perturbational approach, a method that detects large-scale activations that are intrinsically causal. Unlike other measures of complexity that are commonly applied to spontaneous brain signals, PCI accounts only for the information that is generated through deterministic interactions within the thalamocortical system. In this way, the resulting complexity is not affected by random processes, such as noise and muscle activity, or by patterns that are not genuinely integrated, such as the ones generated by isolated neuronal sources or common drivers. Most important, PCI establishes a reliable measurement scale by defining a range of values between various conditions in which consciousness is known to be present (wakefulness, dreaming, LIS), and absent (NREM sleep, different types of anesthesia, stable diagnosis of VS/UWS).

Finally, another clear advantage of this metric is the fact that it can be assessed on the basis of the complexity of cortical interactions, thus independent of the subjects' capacity or willingness to react to external stimuli/commands.

\section{Toward an Understanding of the Neurophysiological Mechanisms Underlying Loss and Recovery of Consciousness}

In addition to improving our ability to detect the presence of conscious experience, linking consciousness to brain complexity - in both theory and practice - may also shed new light on the cortical mechanisms of loss and recovery of consciousness in pathological conditions. Why do complex, long-range cortical interactions collapse into a simple response whenever consciousness is lost?

The striking similarity between TMS-evoked EEG responses during sleep, under anesthesia, and in VS/UWS patients suggests common neuronal mechanisms for LOC in these conditions. In all cases, the complex TMS-evoked activation observed during wakefulness is replaced by a stereotypical positive-negative deflection, which, when TMS is delivered at high intensities, evolves into a graphoelement that matches the EEG criteria for a sleep slow wave, or a K-complex. ${ }^{13}$ Animal $^{31}$ and human ${ }^{32}$ intracranial recordings have shown that both spontaneous EEG sleep slow waves and K-complexes are underpinned by the occurrence of a silent, hyperpolarized down-state in cortical neurons, which is preceded and followed by a period of activation (up-state). This bimodal alternation between up- and down-states reflects an intrinsic bistability in thalamocortical circuits that is thought to depend on neuronal as well as network properties. ${ }^{33-36}$ During NREM sleep, bistability may be mainly caused by increased activity of leak $\mathrm{K}^{+}$ channels, brought about by decreased brainstem cholinergic activity. ${ }^{37}$ Inhalational anesthetics, including nitrous oxide and isoflurane, which strongly potentiate the activity of 2 pore $\mathrm{K}^{+}$ channels, ${ }^{38}$ may act through a similar mechanism. On the other hand, increased inhibition within thalamocortical networks may play a crucial role in inducing bistability ${ }^{36}$ in the case of other anesthetic agents, which act primarily (such as propofol or etomidate), or exclusively (such as midazolam at anesthetic doses) on GABA receptors. 
Our proposition is that, due to bistability, portions of the thalamocortical system, which are otherwise healthy, would not be able to sustain balanced patterns of activations; thus, the inescapable occurrence of a stereotypical down-state after an initial activation would prevent the emergence of complex, long-range patterns of activation in response to a direct stimulation.

This occurrence is particularly relevant for brain-injured patients. As an example, a direct lesion of brainstem activating systems may cause bistability through the very same mechanisms governing NREM sleep. Specifically, brainstem lesions that reduce the cholinergic, noradrenergic, histaminergic and glutamatergic ascending drive may result in enhanced leak and depolarization dependent $\mathrm{K}^{+}$currents in cortical neurons. ${ }^{37}$ Localized alterations, such as undetected cortical epileptic foci, can also exert a strong inhibitory drive on brainstem activating systems, thus producing diffuse cortical bistability. ${ }^{39}$

Alternatively, a form of bistability similar to the one observed during midazolam-induced LOC may result from cortical and subcortical lesions that alter the cortical balance between excitation and inhibition in favor of inhibition. For instance, recovery of language and motor function after stroke can be blocked by excessive inhibitory activity in the perilesional area; ${ }^{40}$ this excessive inhibition may be generated locally or may be projected by healthy areas that become hyperactive. ${ }^{41}$ Thus, cortical lesions that, by themselves, would not necessarily impair consciousness may induce LOC by causing a general unbalance between excitation and inhibition in healthy portions of the thalamocortical system. An excess of thalamocortical inhibition from a hyperactive, subcortical inhibitory area could also explain the paradoxical effects of the sedative zolpidem, a nonbenzodiazepine hypnotic that potentiates $\mathrm{GABA}_{\mathrm{A}}$ receptors, on behavioral improvement of alertness and interactive behavior in severely brain-injured patients. ${ }^{42-44}$ Another crucial event that may induce bistability following brain injury is cortical deafferentation. Severing the white matter with a cortical undercut results in slow waves and in a continuous alternation between up- and down-states in the partially deafferented gyrus, even when the animal and the rest of the brain are awake. ${ }^{45}$

In all cases, evaluating the presence of bistability in the cerebral cortex of brain-injured patients is critically important. Indeed, while anatomical lesions and disconnections cannot be reversed, it may still be possible to reduce bistability and functional disconnections by acting pharmacologically on intrinsic neuronal properties.

Experimentally, one should first demonstrate that the slow wave-like graphoelement triggered by TMS during NREM, under anesthesia, and in VS/UWS patients truly reflects a neuronal down-state (ie, a long-lasting period of membrane hyperpolarization). Finally, one should demonstrate the causal role of bistability by showing that a reduction of TMS-triggered hyperpolarization heralds the recovery of consciousness in acute patients evolving from coma to different degrees of clinical recovery, and that this is paralleled by a recovery of

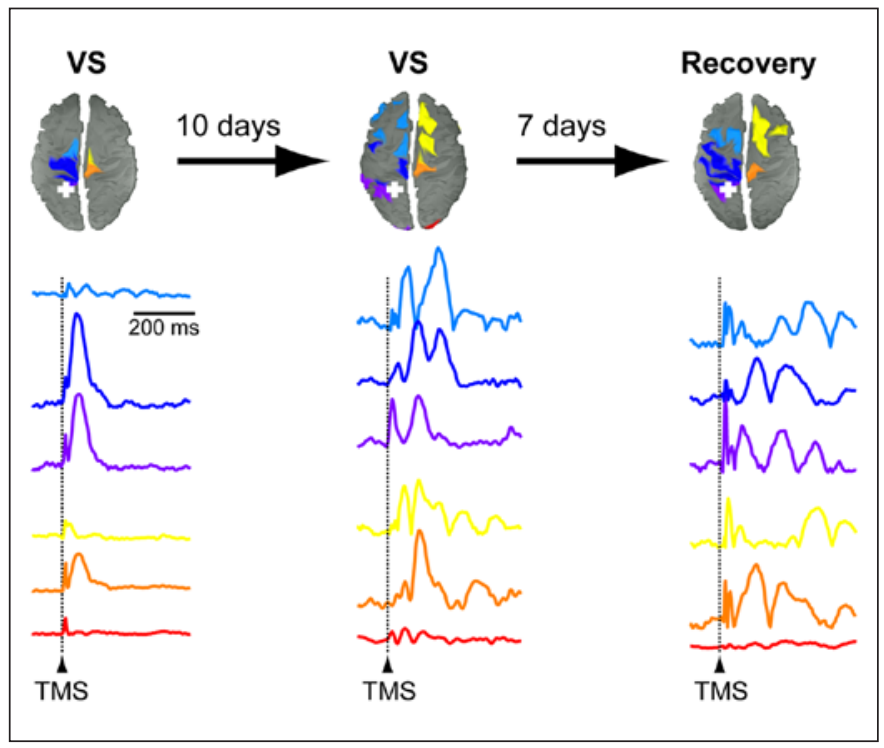

Figure 5. Longitudinal TMS/EEG measurements in brain-injured patients. Recovery of consciousness in a brain-injured patient is accompanied by the recovery of complex EEG activations in response to TMS depicted here at the cortical source level (colored traces). Modified from Rosanova et al. ${ }^{16}$ Interestingly, the regain of EEG complex spatiotemporal dynamics seems to be a stable neurophysiological correlate of consciousness regardless of the behavioral fluctuations characterizing the clinical evolution braininjured patients. The patient here presented was first diagnosed as VS/UWS on the day of the first TMS/EEG measurement (left). Following repeated CRS-R assessments performed every other day, the patient was then clinically assessed as being in a MCS and the second TMS/EEG measurement was planned for the next day. At that time (center) the patient slipped back into a VS/UWS, yet TMS/ EEG responses captured clear-cut signs of long-range, temporally complex dynamics. Within days, the patient was reassessed clinically as being in a MCS and then emerged from MCS on the day of the third TMS/EEG assessment (right) and showed a consolidated complex EEG pattern in response to TMS.

long-range, complex activations at the EEG level, as suggested by our preliminary observation presented in Figure 5 (see also Rosanova et $\mathrm{al}^{16}$ ). Both these observations would strongly suggest that bistability may be the common final pathway for a collapse of brain complexity causing LOC.

Developing and testing noninvasive neurophysiological techniques to these aims is therefore desirable and represents an interesting research goal for future studies. As of today, one of the most promising noninvasive tool to directly test the occurrence of neuronal hyperpolarization associated with a stimulation is represented by the event-related optical signal (EROS), which measures changes in the scattering of nearinfrared light (occurring synchronously with neuronal firing) following a stimulus and combines spatial resolution of few millimeters with temporal resolution in the order of milliseconds. ${ }^{46,47}$ A combined TMS-EROS study has been recently 
published, thus confirming the possibility of simultaneous coregistration. ${ }^{48}$ Future studies should then aim at combining TMS with simultaneous EEG/EROS recordings in acute patients evolving from coma to different degrees of clinical recovery, thus linking the progressive recovery of complexity in the EEG with the vanishing of neuronal hyperpolarization in response to TMS.

\section{Methodological Caveats}

For both the quantification of brain complexity and the understanding of the neurophysiological substrates of the loss and recovery of consciousness, TMS combined with EEG proved to be a reliable tool.

Nonetheless, its application, especially in the context of brain-damaged, noncommunicating patients, raises some caution regarding several methodological aspects. An important caveat is that, in brain-injured patients, the presence of cortical lesions may limit the efficacy of TMS in eliciting a significant cortical response (see, eg, VS/UWS anoxic patients reported in Rosanova et $\mathrm{al}^{16}$ ). For this reason, making sure that TMS perturbation is not applied to a structurally damaged portion of the cortical surface is of paramount importance to gauge reliable responses on which complexity can be properly quantified. This problem can be faced by employing an imaging-guided TMS positioning system to avoid targeting damaged cortical sites. $^{49}$

An associated general issue relates to the handling of scalp and source reconstructed EEG signals in brain-injured patients, often presenting severely deformed scalp/brain geometries. This may lead to imprecise and mislocalized EEG source estimation. The need of accurate head models (via the finite element method) ${ }^{50}$ based on high-resolution magnetic resonance images is therefore key in those cases where accurate cortical localization of the obtained responses is critical. On the other hand, this caveat is less relevant when measuring brain complexity by means of TMS, as an imprecise cortical source localization is not expected to produce changes in the overall complexity of the spatiotemporal dynamics of the response to TMS.

A more direct caveat is that the EEG signal following TMS can be fraught with external sources of noise. In fact, besides the magnetic artifact (attenuated/canceled by the amplifier sample and hold circuit), other factors (if not adequately controlled for) may confound the interpretation of TMS-evoked potentials particularly when performed on noncommunicating brain-injured patients who cannot follow commands. For example, TMS may directly stimulate or activate trigeminal sensory afferents and head muscles, thus evoking somatosensory potentials or muscle potentials, respectively. Scalp muscle artifacts lasting up to $50 \mathrm{~ms}$ (in the form of spikes time-locked with the TMS trigger, with a voltage ranging from hundreds to thousands of $\mu \mathrm{V}^{51}$ ) are recordable mainly in the most lateral and frontal electrodes and can be prevented by stimulating cortical areas underlying scalp regions close to the midline, by changing the coil orientation or by decreasing output level of the stimulator.

At the same time, eye blinks/movements or auditory evoked potentials caused by the "click" associated with the coil's discharge propagating through air and bone may contaminate the genuine cortical response to TMS. ${ }^{52,53}$ The occurrence of these systematic artifacts can be effectively prevented during the experiments by employing noise masking and by adjusting the coil location and the stimulation intensity. ${ }^{12,18,20,21}$

\section{Conclusions}

Here we reviewed several of the relevant aspects for a theorydriven empirical assessment of brain complexity based on the combination of TMS and EEG. The results are encouraging and may be particularly relevant in 2 main aspects.

On one hand, such an approach seems to provide a reliable measuring scale along the unconsciousness/consciousness spectrum. This represents a first step toward a robust and objective assessment of unresponsive individuals whose level of consciousness is unknown.

Most important, a theoretical and empirical link between consciousness and complexity may shed new light on the cortical mechanisms that underlie loss and recovery of consciousness in pathological condition. TMS/EEG measures suggest that also in VS/UWS patients brain complexity may collapse through network bistability, as it does during sleep and anesthesia. Since bistability is, in principle, reversible and its mechanisms are well understood at the cellular and network level, it may represent a suitable target for novel therapeutic approaches in patients in whom consciousness is impaired, in spite of preserved cortical activity.

\section{Declaration of Conflicting Interests}

The author(s) declared no conflicts of interest with respect to the research, authorship, and/or publication of this article.

\section{Funding}

This study was funded by the Belgian National Funds for Scientific Research(FNRS), EU grant FP7-ICT-2011-9, n. 600806 “Corticonics"; PUR 2009, James S. McDonnell Foundation Scholar Award 2013 and "Dote ricerca": FSE, Regione Lombardia (University of Milan); Prin2008 (Italian Government); Fonds Léon Fredericq, James S. McDonnell Foundation; Mind Science Foundation; European Commission (Mindbridge, DISCOS, DECODER \& COST); Concerted Research Action (ARC 06/11-340); and Public Utility Foundation "Université Européenne du Travail" and "Fondazione Europea di Ricerca Biomedica."

\section{References}

1. Tononi G. Consciousness as integrated information: a provisional manifesto. Biol Bull. 2008;215:216-242.

2. Owen AM, Coleman MR, Boly M, Davis MH, Laureys S, Pickard JD. Detecting awareness in the vegetative state. Science. 2006;313:1402-1402. 
3. Cruse D, Chennu S, Chatelle C, et al. Bedside detection of awareness in the vegetative state: a cohort study. Lancet. 2011;378:2088-2094.

4. Blumenfeld H, Taylor J. Why do seizures cause loss of consciousness? Neuroscientist. 2003;9:301-310.

5. Laureys S, Owen AM, Schiff ND. Brain function in coma, vegetative state, and related disorders. Lancet Neurol. 2004;3:537546

6. Arthuis M, Valton L, Régis J, et al. Impaired consciousness during temporal lobe seizures is related to increased long-distance cortical-subcortical synchronization. Brain. 2009;132:2091-2101.

7. Seth AK, Izhikevich E, Reeke GN, Edelman GM. Theories and measures of consciousness: an extended framework. Proc Natl Acad Sci U S A. 2006;103:10799-10804.

8. Sporns O. The human connectome: a complex network. Ann N Y Acad Sci. 2011;1224:109-125.

9. Tononi G, Edelman GM. Consciousness and complexity. Science. 1998;282:1846-1851.

10. Tononi G. An information integration theory of consciousness. BMC Neurosci. 2004;5:42.

11. Massimini M, Boly M, Casali A, Rosanova M, Tononi G. A perturbational approach for evaluating the brain's capacity for consciousness. Prog Brain Res. 2009;177:201-214.

12. Massimini M, Ferrarelli F, Huber R, Esser SK, Singh H, Tononi G. Breakdown of cortical effective connectivity during sleep. Science. 2005;309:2228-2232.

13. Massimini M, Ferrarelli F, Esser SK, et al. Triggering sleep slow waves by transcranial magnetic stimulation. Proc Natl Acad Sci U S A. 2007;104:8496-8501.

14. Massimini M, Ferrarelli F, Murphy M, et al. Cortical reactivity and effective connectivity during REM sleep in humans. Cogn Neurosci. 2010;1:176-183.

15. Ferrarelli F, Massimini M, Sarasso S, et al. Breakdown in cortical effective connectivity during midazolam-induced loss of consciousness. Proc Natl Acad Sci U S A. 2010;107:2681-2686.

16. Rosanova $M$, Gosseries $O$, Casarotto $S$, et al. Recovery of cortical effective connectivity and recovery of consciousness in vegetative patients. Brain. 2012;135:1308-1320.

17. Komssi S, Savolainen P, Heiskala J, Kähkönen S. Excitation threshold of the motor cortex estimated with transcranial magnetic stimulation electroencephalography. Neuroreport. 2007;18:13-16.

18. Casali AG, Casarotto S, Rosanova M, Mariotti M, Massimini M. General indices to characterize the electrical response of the cerebral cortex to TMS. Neuroimage. 2010;49:1459-1468.

19. Virtanen DJ, Ruohonen J, Näätänen R, Ilmoniemi RJ. Instrumentation for the measurement of electric brain responses to transcranial magnetic stimulation. Med Biol Eng Comput. 1999;37:322-326

20. Casarotto S, Romero Lauro LJ, Bellina V, et al. EEG responses to TMS are sensitive to changes in the perturbation parameters and repeatable over time. PLOS ONE. 2010;5:e10281.

21. Rosanova M, Casali A, Bellina V, Resta F, Mariotti M, Massimini M. Natural frequencies of human corticothalamic circuits. $J$ Neurosci. 2009;29:7679-7685.

22. Massimini M, Ferrarelli F, Sarasso S, Tononi G. Cortical mechanisms of loss of consciousness: insight from TMS/EEG studies. Arch Ital Biol. 2012;150:44-55.

23. Giacino JT, Kalmar K, Whyte J. The JFK Coma Recovery ScaleRevised: measurement characteristics and diagnostic utility. Arch Phys Med Rehabil. 2004;85:2020-2029.
24. Ragazzoni A, Pirulli C, Veniero D, et al. Vegetative versus minimally conscious states: a study Using TMS-EEG, sensory and event-related potentials. PLOS ONE. 2013;8:e57069.

25. Seth AK, Barrett AB, Barnett L. Causal density and integrated information as measures of conscious level. Philos Trans A Math Phys Eng Sci. 2011;369:3748-3767.

26. Tononi G, Sporns O, Edelman GM. A measure for brain complexity: relating functional segregation and integration in the nervous system. PNAS. 1994;91:5033-5037.

27. Pincus SM, Gladstone IM, Ehrenkranz RA. A regularity statistic for medical data analysis. J Clin Monit. 1991;7:335-345.

28. Seth AK, Dienes Z, Cleeremans A, Overgaard M, Pessoa L. Measuring consciousness: relating behavioural and neurophysiological approaches. Trends Cogn Sci (Regul Ed). 2008;12:314-321.

29. Casali AG, Gosseries O, Rosanova M, et al. A theoretically based index of consciousness independent of sensory processing and behavior. Sci Transl Med. 2013;5:198ra105-198ra105.

30. Lempel A, Ziv J. On the complexity of finite sequences. IEEE Trans Info Theory. 1976;22:75-81.

31. Steriade M, Timofeev I, Grenier F. Natural waking and sleep states: a view from inside neocortical neurons. J Neurophysiol. 2001;85:1969-1985.

32. Cash SS, Halgren E, Dehghani N, et al. The human K-complex represents an isolated cortical down-state. Science. 2009;324:10841087.

33. Sanchez-Vives MV, McCormick DA. Cellular and network mechanisms of rhythmic recurrent activity in neocortex. Nat Neurosci. 2000;3:1027-1034.

34. Timofeev I, Grenier F, Steriade M. Impact of intrinsic properties and synaptic factors on the activity of neocortical networks in vivo. J Physiol Paris. 2000;94:343-355.

35. Hill S, Tononi G. Modeling sleep and wakefulness in the thalamocortical system. J Neurophysiol. 2005;93:1671-1698.

36. Mann EO, Kohl MM, Paulsen O. Distinct roles of GABA(A) and GABA(B) receptors in balancing and terminating persistent cortical activity. J Neurosci. 2009;29:7513-7518.

37. McCormick DA, Wang Z, Huguenard J. Neurotransmitter control of neocortical neuronal activity and excitability. Cereb Cortex. 1993;3:387-398.

38. Alkire MT, Hudetz AG, Tononi G. Consciousness and anesthesia. Science. 2008;322:876-880.

39. Englot DJ, Yang L, Hamid H, et al. Impaired consciousness in temporal lobe seizures: role of cortical slow activity. Brain. 2010;133:3764-3777.

40. Classen J, Schnitzler A, Binkofski F, et al. The motor syndrome associated with exaggerated inhibition within the primary motor cortex of patients with hemiparetic. Brain. 1997;120(pt 4):605619.

41. Murase N, Duque J, Mazzocchio R, Cohen LG. Influence of interhemispheric interactions on motor function in chronic stroke. Ann Neurol. 2004;55:400-409.

42. Brefel-Courbon C, Payoux P, Ory F, et al. Clinical and imaging evidence of zolpidem effect in hypoxic encephalopathy. Ann Neurol. 2007;62:102-105.

43. Whyte J, Myers R. Incidence of clinically significant responses to zolpidem among patients with disorders of consciousness: a preliminary placebo controlled trial. Am J Phys Med Rehabil. 2009;88:410-418.

44. Schiff ND. Recovery of consciousness after brain injury: a mesocircuit hypothesis. Trends Neurosci. 2010;33:1-9. 
45. Nita DA, Cissé Y, Timofeev I, Steriade M. Waking-sleep modulation of paroxysmal activities induced by partial cortical deafferentation. Cereb Cortex. 2007;17:272-283.

46. Gratton G, Fabiani M. Shedding light on brain function: the eventrelated optical signal. Trends Cogn Sci (Regul Ed). 2001;5:357363.

47. Gratton G, Fabiani M. Fast optical imaging of human brain function. Front Hum Neurosci. 2010;4:52.

48. Parks NA, Maclin EL, Low KA, Beck DM, Fabiani M, Gratton G. Examining cortical dynamics and connectivity with simultaneous single-pulse transcranial magnetic stimulation and fast optical imaging. Neuroimage. 2012;59:2504-2510.

49. Rosanova M, Casarotto S, Pigorini A, Canali P, Casali AG, Massimini M. Combining transcranial magnetic stimulation with electroencephalography to study human cortical excitability and effective connectivity. In: Fellin T, Halassa M, eds. Neuronal Network Analysis. Vol 67. Totowa, NJ: Humana Press; 2011:435-457.

50. Irimia A, Matthew Goh SY, Torgerson CM, Chambers MC, Kikinis R, Van Horn JD. Forward and inverse electroencephalographic modeling in health and in acute traumatic brain injury. Clin Neurophysiol. 2013;124(11):2129-2145.

51. Merletti R, Fiorito A, Lo Conte LR, Cisari C. Repeatability of electrically evoked EMG signals in the human vastus medialis muscle. Muscle Nerve. 1998;21:184-193.

52. Corthout E, Uttl B, Juan CH, Hallett M, Cowey A. Suppression of vision by transcranial magnetic stimulation: a third mechanism. Neuroreport. 2000;11:2345-2349.

53. Nikouline V, Ruohonen J, Ilmoniemi RJ. The role of the coil click in TMS assessed with simultaneous EEG. Clin Neurophysiol. 1999;110:1325-1328. 